\title{
Low nocturnal diastolic ocular perfusion pressure as a risk factor for NTG progression: a 5-year prospective study
}

\author{
Pushpa Raman $^{1} \cdot$ Nurull Bahya Suliman ${ }^{1} \cdot$ Mimiwati Zahari ${ }^{1}{ }^{1}$ Michael Kook $^{2} \cdot$ Norlina Ramli $^{1}$
}

Received: 25 September 2017 / Revised: 22 January 2018 / Accepted: 24 January 2018 / Published online: 1 March 2018

(c) The Royal College of Ophthalmologists 2018

\begin{abstract}
Objective To assess the relationship between baseline intraocular pressure (IOP), blood pressure (BP) and ocular perfusion pressure (OPP), and the 5-year visual field progression in normal-tension glaucoma (NTG) patients.

Design Prospective, longitudinal study.

Methods Sixty-five NTG patients who were followed up for 5 years are included in this study. All the enrolled patients underwent baseline 24-h IOP and BP monitoring via 2-hourly measurements in their habitual position and were followed up for over 5 years with reliable VF tests. Modified Anderson criteria were used to assess VF progression. Univariable and multivariable analyses using Cox's proportional hazards model were used to identify the systemic and clinical risk factors that predict progression. Kaplan-Meier survival analyses were used to compare the time elapsed to confirmed VF progression in the presence or absence of each potential risk factor.

Results At 5-year follow-up, $35.4 \%$ of the enrolled patients demonstrated visual field progression. There were statistically significant differences in the mean diastolic blood pressure $(p<0.05)$ and diastolic OPP $(p<0.05)$ between the progressing and the non-progressing group. There was no association between IOP parameters and NTG progression. Multivariable analysis identified low nocturnal DOPP at baseline as a significant predictive risk factor for glaucomatous visual field progression at 5 years. An mmHg decrease in nocturnal DOPP increases the hazard of progression by 1.4 times. Patients with DOPP $<35 \mathrm{mmHg}$ have 2.3 -fold higher probability of progressing compared to the patients with DOPP $>43.7 \mathrm{mmHg}$ $(\log$ rank $=0.018)$.

Conclusion Diastolic parameters of BP and OPP were significantly lower in the NTG patients who progressed after 5 years. Low nocturnal DOPP is an independent predictor of glaucomatous visual field progression in NTG patients.
\end{abstract}

\section{Introduction}

Normal-tension glaucoma (NTG), also known as lowtension glaucoma, is an optic neuropathy characterized by progressive loss of retinal ganglion cells and their axons, which leads to structural and functional damage to the optic nerve characterised by intraocular pressures (IOPs) lower than $21 \mathrm{mmHg}$ [1]. It is well documented in recent

Norlina Ramli

drnramli@gmail.com

1 Department of Ophthalmology, University of Malaya Eye Research Centre, Faculty of Medicine, University of Malaya, Kuala Lumpur 50603, Malaysia

2 Department of Ophthalmology, College of Medicine, University of Ulsan, Asan Medical Center, Seoul, South Korea literature that alterations in IOP, blood pressure (BP) and ocular perfusion pressure (OPP) can play a significant role in the pathogenesis of the disease [2-5]. Patients with chronically elevated BP or those with significant dips in $\mathrm{BP}$ at night (nocturnal hypotension) may experience dramatic changes in OPP throughout the day. It has been reported that 24-h OPP instability correlates with the incidence and progression of open-angle glaucoma (OAG) [6].

Low diastolic OPP (DOPP) has been strongly associated with the increased prevalence of OAG in the following population-based epidemiological studies: in the Barbados Eye Study, low DOPP $(<55 \mathrm{mmHg})$ was a risk factor for the development of glaucoma, with a relative risk of 3.2 [7]. In another Caucasian-based study, the Baltimore Eye Survey found a six-fold increased OAG prevalence in subjects with low DOPP $(<30 \mathrm{mmHg})$ [8]. The Early Manifest Glaucoma Trial (EMGT) identified that patients with low systolic 
perfusion pressure at baseline progressed faster than their counterparts [9].

Numerous retrospective, cross-sectional studies have been carried out in Asia to establish the relationship between the variability of systemic haemodynamic parameters, OPP, IOP and the incidence of OAG in their local population. The Singapore Malay Eye Study stated that low DBP, low mean OPP (MOPP) and low DOPP are independent risk factors for $\mathrm{OAG}$ development in their ethnic Malays [5]. Choi et al. [2] from South Korea have established that 24-h MOPP instability is a risk factor for the development of NTG and is associated with higher degree of glaucomatous visual field (VF) damage at initial presentation. In a separate retrospective study, Sung et al. [4] also found that greater 24-h MOPP fluctuation is associated with the increased rate of NTG progression. In contrast, Quaranta et al. found no association between $24 \mathrm{~h}$ MOPP and VF damage in Caucasian NTG patients [10].

In view of the higher prevalence of NTG in the Asian population, identifying the factors associated with progression is of particular relevance to combat the burden of this disease [11]. The purpose of this study is to assess prospectively the relationship between baseline 24-h IOP, BP and OPP, and VF progression in NTG patients. We also set out to identify the systemic and ocular risk factors that can predict VF progression in our local population of NTG patients.

\section{Materials and methods}

\section{Subjects}

This study was conducted in the Ophthalmology Department of University Malaya Medical Centre (UMMC) from October 2010 to November 2015. A total of 72 eyes from 72 patients diagnosed with NTG from the University of Malaya Medical Centre eye clinic glaucoma registry were included in the study. Ethics approval was obtained from the Ethics Committee of UMMC and the study adhered to the tenants of the Declaration of Helsinki. Informed consent was obtained from all subjects who agreed to participate in this study.

Patients with NTG from the department glaucoma registry were approached for inclusion in this study. Diagnosis was based on clinical evaluation, which included the presence of glaucomatous optic nerve head damage with corresponding VF loss; a recorded IOP not exceeding 21 $\mathrm{mmHg}$ at any point in clinic using a Goldmann applanation tonometer; open angles on gonioscopy and normal anterior segment structures. Eyes with glaucomatous VF defects were defined as those that met the following criteria confirmed by more than two reliable baseline tests: contiguous non-edge points (allowing the two nasal step edge points) on a Humphrey program 24-2 Swedish Interactive Threshold Algorithm (SITA) standard VF with $p<0.05$ on the total deviation plot, with at least one point at $p<0.01$, and Glaucoma Hemifield Test results outside the normal limits. Reliable VF assessment was defined as a VF test with a false-positive error $<33 \%$, a false-negative error $<33 \%$ and a fixation loss $<20 \%$. The first perimetric result was excluded from analysis, to obviate the learning effects. If both eyes of a patient had NTG and met the inclusion criteria, only the right eye was included in the analysis. Patients who did not complete 5 years follow-up and who were unable to perform reliable Humphrey visual field (HVF) assessment were excluded from the study. Patients with evidence of secondary glaucoma, non-ocular causes of optic nerve damage and history of any other ocular disease that could result in a VF defect were also excluded.

Baseline best-corrected visual acuity was recorded with a LogMar chart. Central corneal thickness (CCT) measurements were recorded in all patients by way of an ultrasound pachymetry device (SP-100 Handy pachymeter, Tomey, Japan). An average of six readings was recorded for each patient. VF examinations were performed with the 24-2 SITA standard strategy on the Humphrey Visual Field Analyser (HFA, Carl-Zeiss Meditec, Dublin, CA).

The methodology for the baseline $24 \mathrm{~h}$ IOP and BP recordings has been elaborated in an earlier publication by Ramli et al. [12]. Briefly, all NTG patients who were on topical IOP-lowering medication underwent a 4-6-week washout period of the anti-glaucoma medications prior to the 24-h IOP measurement and blood pressure monitoring. The washout was done in order to document their actual baseline IOP prior to the treatment and also to rule out those misdiagnosed with high-tension glaucoma. They were restarted on their anti-glaucoma treatments after the 24-h IOP and BP measurements. In order to reflect the actual clinical condition, individuals on systemic antihypertensive or other haemodynamically active medications were not excluded from the study. The 2-hourly IOP and BP measurements were performed-in the sitting position during the diurnal period $(0800-0000 \mathrm{~h})$ and in the supine position during the nocturnal period $(0000-0600 \mathrm{~h})$.

\section{Measurement of haemodynamic parameters}

Mean arterial pressure (MAP) was calculated as follows: $\mathrm{MAP}=\mathrm{DBP}+[1 / 3(\mathrm{SBP}-\mathrm{DBP})] . \mathrm{MAP}$ fluctuation was defined as the difference between the highest and lowest MAPs recorded during the 24-h period. MOPP is expressed as two-thirds of the difference between MAP and IOP, using the formula $\mathrm{MOPP}=2 / 3 \times[\mathrm{DBP}+1 / 3 \times(\mathrm{SBP}-$ DBP)] - IOP. Systolic OPP (SOPP) was calculated as systolic BP-IOP and diastolic OPP (DOPP) was 
calculated as diastolic BP - IOP. Data were organized by three 8-hourly periods, i.e. daytime, evening and nocturnal period.

\section{Determination of VF progression}

Modified Anderson criteria for VF progression were used to determine glaucoma progression throughout the follow-up [13]. Advanced Glaucoma Intervention Study (AGIS) scores were calculated for all patients with NTG from second baseline VFs and were considered to reflect the baseline VF severity. VF progression in our study required an mean deviation on Humphrey visual field printout (MD) deterioration of $>3 \mathrm{~dB}$ compared to the two baseline values, demonstrated at least twice during the follow-up period, and deterioration of three adjacent points in the total deviation plot by $5 \mathrm{~dB}$ or more from the baseline value of each test location, with at least one point depressed by $10 \mathrm{~dB}$ in the early stage of NTG (baseline AGIS score 5 or less). If the baseline AGIS score was over 5, the three adjacent points had to deteriorate $10 \mathrm{~dB}$ or more from the baseline value. Such changes were required to be confirmed in two consecutive perimetric examinations. The time of the first VF progression detection was defined as the end point in a patient showing progression. The mean deviation on Humphrey visual field printout slope was calculated from five consecutive VF measurements. We calculated VF progression rate using linear regression analysis of global perimetric MD values over time, where rate of progression is the slope expressed in $\mathrm{dB} / y e a r$.

\section{Statistical analysis}

All statistical tests were performed using the IBM SPSS for Windows statistical software package (version 20.0; SPSS Inc., Chicago, Illinois, USA). Categorical variables are presented as numbers and percentages, and the $\chi^{2}$-test was used to compare clinical characteristics among the groups. Independent-samples $t$ test and Mann-Whitney $U$ test were used for the analysis of continuous variables when appropriate for the comparisons between the groups.

VF progression analysis included all the follow-up measurements from baseline to the time of progression or last follow-up visit. To identify the risk factors for progression, the IOP, BP and OPP parameters were analysed as continuous variables or as categorical variables by using quartile cut-offs based on the distribution of the study population. Hazard ratios (HRs) for associations between the potential risk factors and VF progression were obtained using Cox's proportional hazards models.

Results were expressed as HR with $95 \%$ confidence intervals (CIs). Univariable analyses were performed separately for each variable. Variables with a probability value
$<0.20$ in univariable analysis were considered significant and were included in a multivariable Cox proportional hazards model.

A backward elimination process was used to develop the final multivariable model. These HRs estimate the effect of a specific factor on the risk of NTG progression in the study while controlling for other significant variables. Interpretation of the HRs should consider that their numerical values represent summary, group-based estimates of relative risk for a given factor.

Kaplan-Meier survival analyses were used to compare the survival experience (time to confirmed VF progression) in the presence or absence of a potential risk factor. DOPP was analysed according to quartiles for this analysis.

\section{Results}

Out of the 72 patients recruited at baseline, 65 completed the minimum 5 years of follow-up. Five patients passed away and two patients were uncontactable. This cohort comprised 33 females (50.8\%) and 32 males (49.2\%), who were included in the final analysis. The mean age at the follow-up examination was $68.2 \pm 9.8$ years (range $47-90$ years). The mean follow-up time was $6.0 \pm 0.7$ years. Mean $\mathrm{VF}$ assessment per patient at the end of the 5-year follow-up period was 10 .

Twenty-three eyes $(35.4 \%)$ reached the progression end point in the 5-year follow-up. The mean rate of global VF change for the entire population was $-0.70 \pm 1.17 \mathrm{~dB} /$ year.

Among the 72 eyes at baseline, $77.8 \%$ were treated with prostaglandin analogue alone, $11.2 \%$ by prostaglandin analogue/topical beta blocker combination, $7 \%$ on prostaglandin analogue/dorzolamide combination, $3 \%$ on topical beta blocker monotherapy and $2 \%$ on brimonidine alone. At the end of the 5-year follow-up, $65 \%$ were on prostaglandin analogue monotherapy, $23 \%$ on dual therapy topical medication, $9 \%$ on triple therapy topical medication and the remaining $3 \%$ were on quadruple topical medications. None of the patients underwent surgical or laser treatment during the follow-up period.

The demographics of the study patients in the progressive and non-progressive groups are summarised in Table 1. There is almost equal distribution among gender in both the groups. Neither group had significant differences in co-morbidities such as hypertension and diabetes. All the hypertensive patients were on combination antihypertensives such as ACE inhibitors, beta blockers, calcium channel blocker and diuretics. Each class of medications was included in the risk analysis. There was no significant relationship found between the class of hypertensive medication and NTG progression. There is a difference in baseline MD between the progressing and non- 
Table 1 Baseline demographic and ophthalmic parameters between progressors and non-progressors

\begin{tabular}{|c|c|c|c|}
\hline & $\begin{array}{l}\text { Progressors } \\
(n=23)\end{array}$ & $\begin{array}{l}\text { Non-progressors } \\
(n=42)\end{array}$ & $p$ Value \\
\hline Mean age \pm SD & $68.96 \pm 9.64$ & $67.61 \pm 10.05$ & 0.630 \\
\hline $\begin{array}{l}\text { Gender: female } \\
(\%)\end{array}$ & $15(45.5 \%)$ & $18(55.5 \%)$ & \\
\hline Hypertension (\%) & $14(32.6 \%)$ & $29(67.4 \%)$ & 0.505 \\
\hline $\begin{array}{l}\text { Diabetes mellitus } \\
(\%)\end{array}$ & $9(34.6 \%)$ & $17(65.4 \%)$ & 0.916 \\
\hline Asthma (\%) & $2(33.3 \%)$ & $4(66.7 \%)$ & 0.912 \\
\hline $\begin{array}{l}\text { LogMAR BCVA } \\
\pm \text { SD }\end{array}$ & $0.30 \pm 0.24$ & $0.19 \pm 0.18$ & 0.780 \\
\hline $\mathrm{CCT}(\mu \mathrm{m}) \pm \mathrm{SD}$ & $0.51 \pm .041$ & $0.50 \pm .036$ & 0.355 \\
\hline Baseline $\mathrm{MD} \pm \mathrm{SD}$ & $-7.43 \pm 5.30$ & $-5.36 \pm 4.92$ & $0.04 *$ \\
\hline Baseline $\mathrm{PSD} \pm \mathrm{SD}$ & $5.64 \pm 3.35$ & $4.20 \pm 2.93$ & 0.62 \\
\hline $\begin{array}{l}5 \text {-Year MD }(\mathrm{dB}) \pm \\
\text { SD }\end{array}$ & $-12.52 \pm 6.58$ & $-6.04 \pm 4.77$ & $<0.001 *$ \\
\hline 5 -Year PSD \pm SD & $7.13 \pm 2.67$ & $4.37 \pm 2.97$ & $<0.001 *$ \\
\hline MD slope \pm SD & $-1.83 \pm 1.09$ & $-0.07 \pm 0.69$ & $<0.001 *$ \\
\hline
\end{tabular}

$B C V A$ best-corrected visual acuity, $C C T$ central corneal thickness, $M D$ mean deviation on Humphrey visual field printout, PSD pattern standard deviation

$* p$ value $<0.05$ is significant

progressing groups $(p=0.04)$, but no significant difference in baseline pattern standard deviation (PSD) $(p=0.62)$. At baseline, 41 patients $(63.1 \%)$ were in the early stage of NTG (AGIS score <5). The mean baseline AGIS score in the progressor and non-progressor groups was 5.7 and 4.6, respectively. This difference was not statistically significant $(p=0.37)$.

Systemic haemodynamic parameters are shown in Table 2. The progressing group had significantly lower diastolic pressure and diastolic perfusion pressure throughout the day, evening and night-time compared to the non-progressing group. There was no significant difference detected in the IOP parameters throughout the 24-h measurements (Table 3). Our study also did not reveal any significant difference in the nocturnal dip percentage in the progressing group. Progressing eyes and stable eyes had a mean global rate of VF change of $-1.86 \pm 1.06$ and -0.07 $\pm 0.61 \mathrm{~dB} /$ year, respectively $(p<0.001)$.

Univariable analysis was carried out on all the demographic factors, IOP, BP, OPP and VF parameters to identify the risk factors associated with progression in this cohort of NTG patients. The results in Table 4 summarise the significant factors in the univariable analysis $(p<0.20)$ from IOP parameters, BP and OPP parameters and VF parameters. Multivariable analysis shows that low nocturnal DOPP at baseline is associated with significantly higher risk of glaucomatous VF progression at 5 years. A unit $(\mathrm{mmHg})$ decrease in DOPP at night increases the hazard of
Table 2 Systemic blood pressure measurement difference between progressors and non-progressors

\begin{tabular}{|c|c|c|c|}
\hline & $\begin{array}{l}\text { Progressors } \\
(n=23)\end{array}$ & $\begin{array}{l}\text { Non-progressors } \\
(n=42)\end{array}$ & $p$ Value \\
\hline Mean SBP day & $133.55 \pm 15.89$ & $134.72 \pm 16.62$ & 0.752 \\
\hline $\begin{array}{l}\text { Mean SBP } \\
\text { evening }\end{array}$ & $132.11 \pm 19.67$ & $132.69 \pm 27.53$ & 0.537 \\
\hline Mean SBP night & $123.40 \pm 22.16$ & $122.80 \pm 16.11$ & 0.752 \\
\hline Mean DBP day & $75.90 \pm 10.16$ & $80.35 \pm 8.94$ & $0.038^{*}$ \\
\hline $\begin{array}{l}\text { Mean DBP } \\
\text { evening }\end{array}$ & $74.54 \pm 6.90$ & $80.37 \pm 8.75$ & $0.008^{*}$ \\
\hline $\begin{array}{l}\text { Mean DBP } \\
\text { night }\end{array}$ & $70.30 \pm 9.58$ & $75.82 \pm 6.82$ & $0.009 *$ \\
\hline MAP day & $95.11 \pm 8.74$ & $99.55 \pm 11.84$ & 0.120 \\
\hline MAP evening & $93.74 \pm 8.20$ & $97.89 \pm 12.02$ & 0.145 \\
\hline MAP night & $88.00 \pm 11.83$ & $91.25 \pm 9.03$ & 0.220 \\
\hline Nocturnal dip & $5.49 \pm 7.65$ & $6.91 \pm 8.77$ & 0.516 \\
\hline $\begin{array}{l}\text { Nocturnal dip } \\
(\%)\end{array}$ & $8.21 \pm 11.47$ & $8.67 \pm 10.93$ & 0.874 \\
\hline
\end{tabular}

Continuous variables are expressed as mean \pm standard deviation. Independent $t$ test or Mann-Whitney $U$ test is used to detect the differences between groups

$S B P$ systolic blood pressure, $D B P$ diastolic blood pressure, $M A P$ mean arterial pressure

${ }^{*} p$ value $<0.05$ is significant

progression by 1.4 times. Among the VF values, baseline PSD is a significant predictor for progression. A unit increase in baseline PSD $(\mathrm{dB})$ increases the hazard of progression by $14 \%$.

Kaplan-Meier survival analysis was used to estimate the probability of non-progression of VF loss at the 5-year follow-up visit (Fig. 1). The cumulative probability of nonprogression of VF loss was $38 \%$ at 60 months in patients with DOPP $<35 \mathrm{mmHg}$ and $88 \%$ in those with DOPP $>$ $43.7 \mathrm{mmHg}$. This between-group difference was significant ( $p=0.018$, log-rank test). Thus, patients with DOPP $<35$ $\mathrm{mmHg}$ have 2.3-fold higher probability of progressing compared to the patients with DOPP $>43.7 \mathrm{mmHg}$.

\section{Discussion}

This prospective study shows that systemic parameters such as ocular perfusion pressure are an important factor for progression among NTG patients. Furthermore, it also shows that intraocular pressure and its fluctuation is not a significant factor in the progression of this disease, unlike in high-tension glaucoma.

In this study, we have found that $35.4 \%$ of the NTG patients progressed despite IOP-lowering treatments. This 
Table 3 Ophthalmic measurements' difference between progressors and non-progressors

\begin{tabular}{|c|c|c|c|}
\hline & $\begin{array}{l}\text { Progressors } \\
(n=23)\end{array}$ & $\begin{array}{l}\text { Non-progressors } \\
(n=42)\end{array}$ & $p$ Value \\
\hline \multicolumn{4}{|l|}{ IOP parameters } \\
\hline Mean IOP day & $15.01 \pm 2.47$ & $14.78 \pm 2.07$ & 0.688 \\
\hline $\begin{array}{l}\text { Mean IOP } \\
\text { evening }\end{array}$ & $14.35 \pm 2.77$ & $14.24 \pm 2.16$ & 0.863 \\
\hline Mean IOP night & $15.55 \pm 2.10$ & $14.99 \pm 1.91$ & 0.278 \\
\hline IOP fluctuation & $6.60 \pm 1.20$ & $6.99 \pm 1.70$ & 0.328 \\
\hline \multicolumn{4}{|l|}{ OPP parameters } \\
\hline SOPP day & $79.03 \pm 11.21$ & $80.60 \pm 10.73$ & 0.580 \\
\hline SOPP evening & $78.52 \pm 13.50$ & $80.56 \pm 11.74$ & 0.414 \\
\hline SOPP night & $71.91 \pm 15.12$ & $71.94 \pm 10.71$ & 0.510 \\
\hline $\begin{array}{c}\text { SOPP } \\
\text { fluctuation }\end{array}$ & $11.41 \pm 5.70$ & $12.84 \pm 9.19$ & 0.869 \\
\hline DOPP day & $40.58 \pm 6.64$ & $43.71 \pm 5.51$ & $0.047 *$ \\
\hline DOPP evening & $40.13 \pm 4.40$ & $44.08 \pm 5.58$ & $0.050 *$ \\
\hline DOPP night & $36.50 \pm 7.07$ & $40.61 \pm 4.58$ & $0.006^{*}$ \\
\hline $\begin{array}{l}\text { DOPP } \\
\text { fluctuation }\end{array}$ & $7.75 \pm 3.88$ & $6.29 \pm 3.47$ & 0.148 \\
\hline MOPP day & $53.40 \pm 6.11$ & $56.39 \pm 7.48$ & 0.106 \\
\hline MOPP evening & $52.93 \pm 5.68$ & $56.24 \pm 6.91$ & 0.054 \\
\hline MOPP night & $47.72 \pm 9.39$ & $50.85 \pm 6.13$ & 0.110 \\
\hline $\begin{array}{l}\text { MOPP } \\
\text { fluctuation }\end{array}$ & $9.06 \pm 4.96$ & $8.67 \pm 5.22$ & 0.770 \\
\hline
\end{tabular}

Continuous variables are expressed as mean \pm standard deviation. Independent $t$-test or Mann-Whitney $U$ test is used to detect the differences between groups

$I O P$ intraocular pressure, $O P P$ ocular perfusion pressure, SOPP systolic ocular perfusion pressure, $D O P P$ diastolic ocular perfusion pressure, $M O P P$ mean ocular perfusion pressure

$* p$ value $<0.05$ is significant

number is higher than that found by the Collaborative Normal Tension Glaucoma Study (CNTGS), which reported $12 \%$ progression in their treatment arm [14]. However, this figure is debatable since the overall intent-to-treat survival analysis showed no significant difference between the VF progression between the treated and the untreated control groups, owing to the incident of cataract formation [15]. Similar figures for progression have been reported by Sung et al. in the Korean population, where the progression percentage ranged from 33 to $37.6 \%$ in their 4-6-year follow-up study [4]. A long-term evaluation study by Wills Eye Hospital, USA, reported that about $50 \%$ of their NTG patients showed glaucoma progression after $>8$ years despite treatment [16]. We also reported a higher rate of progression of $-0.70 \mathrm{~dB} /$ year in patients with normaltension glaucoma. The average rate of $\mathrm{VF}$ progression in eyes with untreated NTG was reported to be $-0.43 \mathrm{~dB} /$ year in the CNTGS study and $-0.36 \mathrm{~dB} /$ year in the EMGT study. The difference in rates of progression suggests that
Table 4 Univariate and multivariate Cox proportional hazards model with backward elimination for the prediction of visual field progression in NTG patients

\begin{tabular}{|c|c|c|c|c|}
\hline \multirow[t]{2}{*}{$\begin{array}{l}\text { Age and CCT } \\
\text { controlled }\end{array}$} & \multicolumn{2}{|c|}{$\begin{array}{l}\text { Univariate regression } \\
\text { analysis }^{\mathrm{a}}\end{array}$} & \multicolumn{2}{|c|}{$\begin{array}{l}\text { Multivariate Regression } \\
\text { Analysis }\end{array}$} \\
\hline & HR (95\% CI) & $p$ value & HR $(95 \%$ CI $)$ & $p$ value \\
\hline $\begin{array}{l}\text { Mean DBP } \\
\text { day }\end{array}$ & $0.97(0.93-1.01)$ & 0.103 & & \\
\hline $\begin{array}{l}\text { Mean DBP } \\
\text { evening }\end{array}$ & $0.94(0.91-0.99)$ & $0.016^{\mathrm{b}}$ & & \\
\hline $\begin{array}{l}\text { Mean DBP } \\
\text { night }\end{array}$ & $0.94(0.89-0.99)$ & $0.017^{\mathrm{b}}$ & & \\
\hline MAP day & $0.97(0.93-1.01)$ & 0.172 & & \\
\hline MAP night & $0.98(0.94-1.01)$ & 0.183 & & \\
\hline DOPP day & $0.94(0.89-1.01)$ & 0.069 & & \\
\hline $\begin{array}{l}\text { DOPP } \\
\text { evening }\end{array}$ & $0.91(0.84-0.98)$ & $0.010^{\mathrm{b}}$ & & \\
\hline DOPP night & $0.91(0.85-0.98)$ & $0.011^{\mathrm{b}}$ & $\begin{array}{l}0.71 \\
(0.55-0.93)\end{array}$ & $0.012^{\mathrm{b}}$ \\
\hline MOPP day & $0.95(0.89-1.02)$ & 0.153 & & \\
\hline $\begin{array}{l}\text { MOPP } \\
\text { evening }\end{array}$ & $0.94(0.88-1.01)$ & 0.071 & & \\
\hline MOPP night & $0.95(0.89-1.01)$ & 0.111 & & \\
\hline Baseline MD & $0.95(0.89-1.02)$ & 0.153 & & \\
\hline Baseline PSD & $1.10(0.98-1.23)$ & 0.120 & $\begin{array}{l}1.14 \\
(1.00-1.29)\end{array}$ & $0.050^{\mathrm{b}}$ \\
\hline $\begin{array}{l}\text { Central VF } \\
\text { defect }^{c}\end{array}$ & $\begin{array}{l}2.534 \\
(1.108-5.794)\end{array}$ & $0.028^{\mathrm{b}}$ & $\begin{array}{l}3.56 \\
(1.17-10.82)\end{array}$ & $0.025^{\mathrm{b}}$ \\
\hline
\end{tabular}

${ }^{\mathrm{a}}$ For Univariate only parameters with $p<0.2$ are shown on table

${ }^{\mathrm{b}} p$ values $<0.05$ are statistically significant

${ }^{\mathrm{c}}$ Peripheral VF defect used as reference category

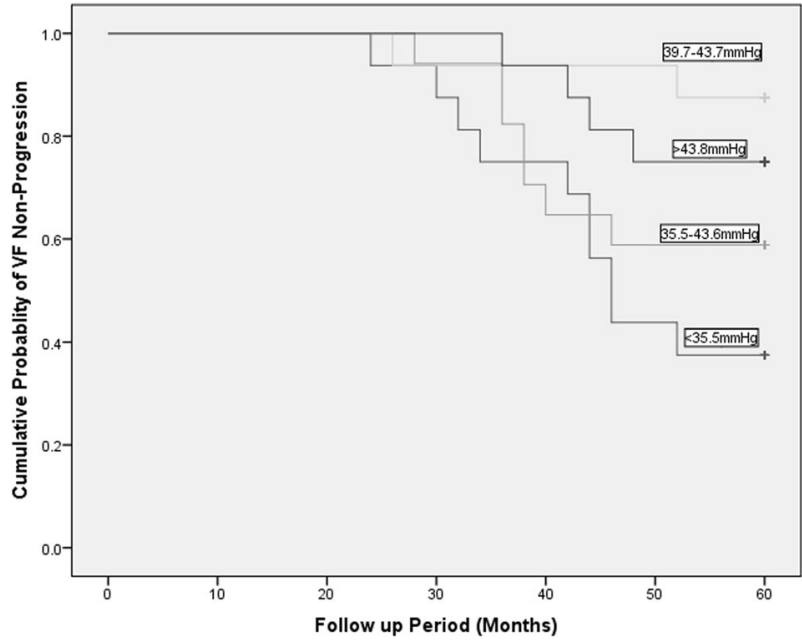

Fig. 1 Survival analysis for nocturnal DOPP. Kaplan-Meier survival analysis to compare the survival experience (time to confirmed glaucomatous visual field progression) for quartile groups of nocturnal DOPP 
NTG in the Asian population is a more severe disease compared to the Western population. Factors supporting this are beyond the scope of this study.

In our aim to identify the relationship between circadian variability in IOP, BP and OPP parameters, and glaucomatous visual progression, we have found that nocturnal events are the main key players that influence the disease process. Similarly, Charlson et al. [17] showed that cumulative nocturnal hypotension predicted VF loss, suggesting that the duration and magnitude of decrease in nocturnal BP below the daytime mean BP, especially pressures that are $10 \mathrm{mmHg}$ lower than daytime mean BP, predict the progression of NTG. While other studies were focusing on daytime and night-time variability in the hemodynamic parameters, we analysed the 24 -h variability by averaging the 2 hourly results into day, evening and night subgroups. According to our analysis, daytime and evening IOP, BP and OPP parameters are not significant factors for VF progression in NTG patients. However, glaucomatous VF progression is significantly associated with nocturnal measurements, such as low nocturnal OPP due to low systemic blood pressure and/or elevated nocturnal IOP. In our study, DOPP at night emerged as a major risk factor that predisposes NTG patients for VF progression. A $1 \mathrm{mmHg}$ decrease in DOPP increases the hazard of progression by 1.4 times. Patients with DOPP $<35 \mathrm{mmHg}$ have 2.3 -fold higher probability of progressing compared to the patients with DOPP in the higher quartile.

Low DOPP has been strongly associated with increased prevalence of OAG in multiple population-based epidemiological studies. In the Barbados Eye Study, low DOPP $(<55 \mathrm{mmHg})$ was a risk factor for the development of glaucoma, with a relative risk of 3.2. After 9 years of further follow-up, it was shown that individuals with the lowest $20 \%$ of DOPP were 2.2 times more likely to develop glaucoma [7]. In another Caucasian-based study, the Baltimore Eye Survey found a six-fold increased OAG prevalence in subjects with low diastolic perfusion pressure $(<30 \mathrm{mmHg})$ [8]. Similarly, the Egna-Neumarkt Study reported a $4.5 \%$ increase in glaucoma prevalence in patients with DOPP $<50 \mathrm{mmHg}$ compared with those with DOPP of $\geq 66 \mathrm{mmHg}$ [18]. In the Proyecto VER study, Quigley et al. also confirmed a four-fold increase in OAG prevalence at DOPP $<50 \mathrm{mmHg}$ [19]. It is worthwhile noting that all these population-based studies only looked at IOP and BP parameters during daytime, unlike our study that looked at these parameters over a 24-h period. Okumura et al. found that low diastolic BP was associated with glaucomatous VF progression in subjects with NTG [20].

Low DOPP is important in the progression of glaucoma, in accordance with the concept that most tissue perfusion occurs during diastole [21]. Our study is the first to report that low nocturnal DOPP is associated with VF progression in NTG patients.

We found that none of the IOP parameters, including the mean IOP in the day, evening and night, and 24-h IOP fluctuation, was associated with the increased risk of global progression. This contradicts the CNTGS and EMGT findings that show that higher follow-up IOP values increase the risk of VF progression in OAG patients [14, 22]. An 8-year follow-up study in Wills Eye Hospital also found that high-peak IOP was a significant risk factor for progression [16]. In our study, patients in both progressing and non-progressing groups maintained a relatively stable IOP (baseline mean IOP: $14.8 \mathrm{mmHg}$ and follow-up mean IOP: $14.1 \mathrm{mmHg}$ ) exhibiting minimal IOP-lowering effect with treatment. This further supports the notion that nonIOP-related risk factors play important role in NTG progression in our local population. NTG is seen more in the elderly people, with a prevalence of $1.6 \%$ in the population over the age of 75 years. However, Anderson et al. have reported that up to $30 \%$ of patients with NTG will be under the age of 50 years [1]. Our study did not find any association between age and increased risk of progression in the NTG patients. A similar finding was documented in CNTGS, where the age did not have any statistically significant relationship with disease progression [14]. Similar to the Low-Pressure Glaucoma Treatment Study (LoGTS), we did not find a significant association between CCT and VF progression [23].

De Moraes et al. demonstrated that lower mean OPP and use of systemic antihypertensives were independently associated with disease progression [24]. However, in our analysis we did not find any association between use of antihypertensive medications and NTG progression.

Several limitations were identified in our study. Only baseline 24-h IOP and BP measurements were included in the analysis. We did not include these measurements at the 5-year endpoint, as the aim was to identify the baseline factors influencing the progression. Calculation of OPP is also hypothetical and may not reflect the true physiological status of ocular perfusion in the optic nerve head. Refractive error was also not recorded in the subjects. This may be a confounding factor in NTG. Lastly, the 30\% reduction in IOP as advocated by CNTGS was not achieved in our progressing group of patients. In this study, the mean IOP of the progressing group at baseline was $15.4 \mathrm{mmHg}$, and at 5year follow-up was $13.3 \mathrm{mmHg}$ (13.6\% reduction). The target IOP that would have been $11 \mathrm{mmHg}$ was not achieved in both the progressing and non-progressing groups. Most patients were on maximal tolerable antiglaucoma medications to ensure good compliance. Two patients who were offered surgical treatment opted to stay on medical treatment. However, the IOP was stable throughout the 5 years of follow-up and all the IOP 
parameters were not related to VF progression in the final analysis.

In conclusion, we have identified that the baseline diastolic blood pressure and diastolic pressure parameters were significant factors in 5-year VF progression among NTG patients. Lower nocturnal DOPP is an independent predictor of glaucomatous VF progression in these patients.

\section{Summary}

\section{What was known before}

- Low DOPP is associated with increased prevalence of open-angle glaucoma.

\section{What this study adds}

- Low nocturnal DOPP is an independent predictor of glaucomatous visual field progression in NTG patients.

\section{Compliance with ethical standards}

Conflict of interest The authors declare that they have no conflict of interest.

\section{References}

1. Anderson DR. Normal-tension glaucoma (low-tension glaucoma). Indian J Ophthalmol. 2011;59(Suppl):S97-101.

2. Choi J, Kim KH, Jeong J, Cho HS, Lee CH, Kook MS. Circadian fluctuation of mean ocular perfusion pressure is a consistent risk factor for normal-tension glaucoma. Invest Ophthalmol Vis Sci. 2007;48:104-11.

3. Joe SG, Choi J, Sung KR, Park SB, Kook MS. Twenty-four hour blood pressure pattern in patients with normal tension glaucoma in the habitual position. Korean J Ophthalmol. 2009;23:32-9.

4. Sung KR, Lee S, Park SB, Choi J, Kim ST, Yun SC, et al. Twenty-four hour ocular perfusion pressure fluctuation and risk of normal-tension glaucoma progression. Invest Ophthalmol Vis Sci. 2009;50:5266-74.

5. Zheng Y, Wong TY, Mitchell P, Friedman DS, He M, Aung T. Distribution of ocular perfusion pressure and its relationship with open-angle glaucoma: the singapore malay eye study. Invest Ophthalmol Vis Sci. 2010;51:3399-404.

6. Chan KK-W, Ho JC-H, Leung DY-L, Ko ST-C. Ocular perfusion pressure and glaucoma: a review. Hong Kong J Ophthalmol. 2016;20:19-28.

7. Leske MC, Wu S-Y, Hennis A, Honkanen R, Nemesure B, Group BS. Risk factors for incident open-angle glaucoma: the Barbados Eye Studies. Ophthalmology. 2008;115:85-93.
8. Tielsch JM, Sommer A, Katz J, Royall RM, Quigley HA, Javitt J. Racial variations in the prevalence of primary open-angle glaucoma: the Baltimore Eye Survey. JAMA. 1991;266:369-74.

9. Heijl A, Bengtsson B, Chauhan BC, Lieberman MF, Cunliffe I, Hyman L, et al. A comparison of visual field progression criteria of 3 major glaucoma trials in early manifest glaucoma trial patients. Ophthalmology. 2008;115:1557-65.

10. Quaranta L, Katsanos A, Riva I, Dastiridou A, Oddone F, Roberti $\mathrm{G}$, et al. Twenty-four-hour intraocular pressure and ocular perfusion pressure characteristics in newly diagnosed patients with normal tension glaucoma. Eye. 2016;30:1481-9.

11. Cho HK, Kee C. Population-based glaucoma prevalence studies in Asians. Surv Ophthalmol. 2014;59:434-47.

12. Ramli N, Nurull B, Hairi NN, Mimiwati Z. Low nocturnal ocular perfusion pressure as a risk factor for normal tension glaucoma. Prev Med. 2013;57:S47-S9.

13. Anderson DR, Patella VM. Automated static perimetry. St. Louis: Mosby; 1999.

14. Anderson DR, Normal Tension Glaucoma Study. Collaborative normal tension glaucoma study. Curr Opin Ophthalmol. 2003;14:86-90.

15. Anderson DR, Drance SM, Schulzer M. The effectiveness of intraocular pressure reduction in the treatment of normal-tension glaucoma. Collaborative Normal-Tension Glaucoma Study Group. Am J Ophthalmol. 1998;126:498-505.

16. Erdem E, Williams A, Kuchar SD, Waisbourd M, Spaeth GL. Long-term ( $>8$ years) evaluation of progression in patients with low-pressure glaucoma. Eur J Ophthalmol. 2015;25:490-5.

17. Charlson ME, de Moraes CG, Link A, Wells MT, Harmon G, Peterson JC, et al. Nocturnal systemic hypotension increases the risk of glaucoma progression. Ophthalmology. 2014;121:2004-12.

18. Bonomi L, Marchini G, Marraffa M, Bernardi P, De Franco I, Perfetti S, et al. Prevalence of glaucoma and intraocular pressure distribution in a defined population: the Egna-Neumarkt Study. Ophthalmology. 1998;105:209-15.

19. Quigley HA, West SK, Rodriguez J, Munoz B, Klein R, Snyder R. The prevalence of glaucoma in a population-based study of Hispanic subjects: Proyecto VER. Arch Ophthalmol. 2001;119:1819-26.

20. Okumura Y, Yuki K, Tsubota K. Low diastolic blood pressure is associated with the progression of normal-tension glaucoma. Ophthalmologica. 2012;228:36-41.

21. Flammer J, Orgül S, Costa VP, Orzalesi N, Krieglstein GK, Serra LM, et al. The impact of ocular blood flow in glaucoma. Progress Retin Eye Res. 2002;21:359-93.

22. Leske MC, Heijl A, Hussein M, Bengtsson B, Hyman L, Komaroff E, et al. Factors for glaucoma progression and the effect of treatment: the early manifest glaucoma trial. Arch Ophthalmol. 2003;121:48-56.

23. Krupin T, Liebmann JM, Greenfield DS, Ritch R, Gardiner S, Low-Pressure Glaucoma Study Group. A randomized trial of brimonidine versus timolol in preserving visual function: results from the Low-Pressure Glaucoma Treatment Study. Am J Ophthalmol. 2011;151:671-81.

24. De Moraes CG, Liebmann JM, Greenfield DS, Gardiner SK, Ritch $\mathrm{R}$, Krupin T, et al. Risk factors for visual field progression in the low-pressure glaucoma treatment study. Am J Ophthalmol. 2012;154:702-11. 